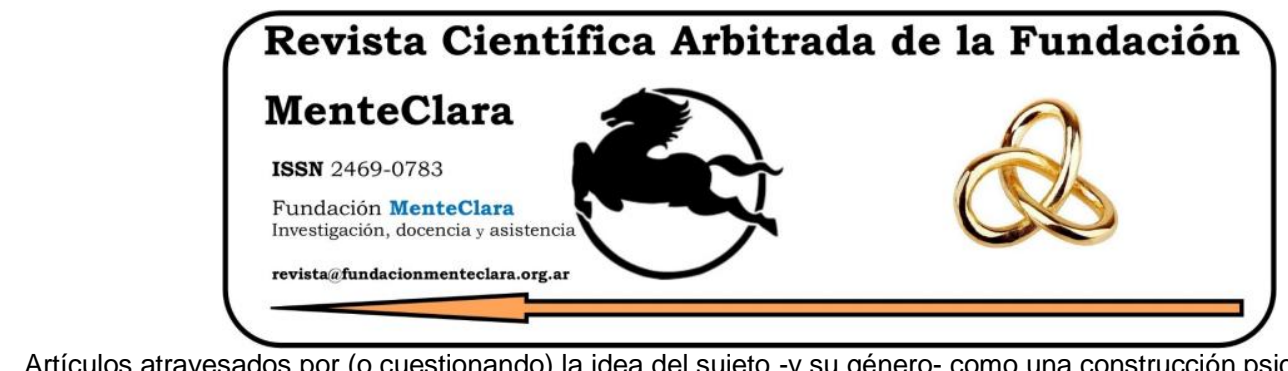

Artículos atravesados por (o cuestionando) la idea del sujeto -y su género- como una construcción psicobiológica de la cultura. Articles driven by (or questioning) the idea of the subject -and their gender- as a cultural psychobiological construction.

Vol. 5 (2020), enero-diciembre ISSN 2469-0783

https://datahub.io/dataset/2020-5-e202

\title{
ESTRATEGIAS DE AFRONTAMIENTO Y SU ROL INTERMEDIARIO FRENTE AL ESTRÉS FAMILIAR. REVISIÓN BIBLIOGRÁFICA
}

\author{
COPING STRATEGIES AND THEIR INTERMEDIARY ROLE IN FAMILY STRESS. A \\ BIBLIOGRAPHIC REVIEW
}

Martín Leoncio de Reyes Balboa martin.balboa@uflouniversidad.edu.ar Facultad de Psicología y Ciencias Sociales, Universidad de Flores, Argentina

Cómo citar este artículo / Citation: Balboa, M. (2020). Estrategias de afrontamiento y su rol intermediario frente al estrés familiar. Revisión bibliográfica. Revista Científica Arbitrada de la Fundación MenteClara, Vol.5 (202). DOI:

https://doi.org/10.32351/rca.v5.202

Copyright: (C) 2020 RCAFMC. Este artículo de acceso abierto es distribuido bajo los términos de la licencia Creative Commons Attribution 4.0 International License (CC BY 4.0). Recibido: 29/11/2020. Aceptado: 07/12/2020 Publicación online: 10/12/2020

Conflicto de intereses: Ninguno que declarar.

\section{Resumen}

En el presente trabajo encontraremos, a partir de una revisión bibliográfica sobre artículos académicos seleccionados de la última década, la manera en que las estrategias de afrontamiento aparecen como variables significativas en problemáticas familiares típicas relacionadas a la pareja, la violencia intrafamiliar, la crianza y el divorcio, intermediando entre los eventos estresantes y su impacto sobre el bienestar del sistema familiar. Se concluye que las estrategias de afrontamiento median entre los estresores y la salud de la familia como grupo y de sus integrantes individualmente.

\begin{abstract}
In the present work we will find, based on a bibliographic review on selected academic articles from the last decade, the way in which coping strategies appear as significant variables in typical family problems related to the couple, domestic violence, parenting and divorce, mediating between stressful events and their impact on the
\end{abstract}


well-being of the family system. It is concluded that coping strategies mediate between stressors and the health of the family as a group and of its members individually.

Palabras Claves: afrontamiento; estrategias de afrontamiento; divorcio; afrontamiento familiar

Keywords: coping; coping strategies; divorce; family coping 


\section{Afrontamiento}

Hay varias definiciones de afrontamiento, pero en general el enfoque transaccional de Lazarus es actualmente aceptado por los expertos en estrategias de afrontamiento.

Lazarus (1966) considera que el afrontamiento es un proceso que se activa cuando una amenaza es percibida, y que apunta a regular los conflictos emocionales y a eliminar esa amenaza.

En estudios posteriores, Lazarus y Folkman (1984) sugieren que hay una relación entre stress y afrontamiento, y este último es considerado la respuesta apropiada al estrés. Por lo tanto, para los autores el afrontamiento se define como los esfuerzos cognitivos y conductuales en constante cambio para gestionar demandas específicas externas y / o internas que son evaluados como cargas o que incluso exceden los recursos de la persona.

En sentido similar, Martínez Montilla y Marín (2017) afirman que el afrontamiento es un proceso dinámico, que se define como el conjunto de recursos que un sujeto utiliza para resolver o mejorar situaciones problemáticas, y reducir las tensiones que esas situaciones generan. Estos recursos pueden ser creencias, motivaciones, habilidades sociales, apoyo social y recursos materiales.

\section{Tipos de estrategias de afrontamiento}

Según Compas (2001), las categorias de afrontamiento más utilizadas son el afrontamiento centrado en el problema y el afrontamiento centrado en las emociones.

El afrontamiento centrado en el problema es cuando un individuo toma medidas para cambiar las circunstancias que causan estrés. 
El afrontamiento centrado en las emociones es cuando un individuo trabaja para modificar su propia experiencia de la emoción negativa que resulta de una fuente de estres.

El afrontamiento por evitación también se ha estudiado como una categoría de afrontamiento y se refiere a las respuestas de afrontamiento que se orientan a alejarse de la fuente del estrés -por ejemplo, retraimiento o negación-.

La amplitud de estas categorias ha llevado a la investigación individual sobre muchos comportamientos de afrontamiento específicos, que incluyen: resolución de problemas, reestructuración cognitiva, catastrofización, actividades físicas, autocrítica, humor, retraimiento social, aceptación resignada, consumo de alcohol o drogas, buscar apoyo social, recurrir a la religión.

Por último, es de notar que según Horwitz (2011), la inconsistencia de los investigadores en como categorizar estos comportamientos específicos impide comparaciones precisas entre los estudios.

\section{Estrategias de afrontamiento en el ámbito de lo familiar}

Martinez Montilla y Marín (2017) extrapolando estos conceptos al ámbito de los sistemas familiares, mencionan el denominado afrontamiento familiar, que es la capacidad de la familia para enfrentarse, movilizarse y poner en acción medidas que actúen sobre las exigencias que demandan cambios o ante la aparición de acontecimientos estresantes.

Las estrategias de afrontamiento familiar pueden fortalecer y preservar los recursos de la familia, y así protegerla de las situaciones estresantes a través de un manejo más adaptativo de la dinámica familiar. Las estrategias de afrontamiento se manifiestan a través de la comunicación, 
de los vínculos y de la promoción de una autoestima positiva entre sus miembros.

Por último, en el presente artículo se realiza una revisión de diversas investigaciones empíricas sobre afrontamiento, articulándolas a partir de la transversalidad de las mismas respecto de problemáticas familiares típicas.

\section{Estrategias de afrontamiento en la pareja}

\section{Afrontamiento diádico}

El ciclo de vida de la familia, en varias de sus configuraciones teóricas, comienza por la formación de la pareja y es en la pareja donde confluyen los modelos de afrontamiento individuales de sus integrantes. Es a través del interjuego de estos modelos del marido y la mujer, que surgen las modalidades específicas de esa nueva familia y que -a través del proceso de crianza- serán transmitidas a los hijos. Para Goleman (1995) la vida en familia es una escuela emocional que opera tanto a través de las cosas que los padres dicen o hacen a los niños, como a través de los modelos que ofrecen para enfrentarse a los sentimientos propios y a los que se dan entre la pareja.

Sin embargo, las estrategias de afrontamiento de la pareja no son solo la suma de las estrategias individuales, sino que deben pensarse en términos diádicos, de afrontamiento relacional.

Es así que la literatura científica comienza a estudiar la manera como las parejas manejan el estrés relacional. En ese sentido, para Papp (2010) el afrontamiento diádico se postula como un importante predictor de cómo las parejas que lidian con problemas crónicos obtienen resultados en términos de resultados de salud, psicosociales y relacionales. 
Sus estudios de funcionamiento relacional revelaron asociaciones entre el afrontamiento individual y el afrontamiento diádico en la dirección del bienestar. Es decir que el afrontamiento diádico tanto negativo como positivo implica contribuciones específicas al bienestar que van más allá de las contribuciones de los estilos de afrontamiento individuales.

\section{Afrontamiento de la Violencia intrafamiliar}

Las estrategias de afrontamiento ante el fenómeno específico de la Violencia Intrafamiliar, ameritan un tratamiento aparte. Mengo (2017) se ocupó específicamente del tema y encontró que si bien el $67.5 \%$ de las mujeres expuestas a violencia intrafamiliar reportaron algún síntoma de salud mental, las estrategias de afrontamiento distinguieron significativamente la experiencia de esos sintomas. Los hallazgos sugieren que disponer de recursos de afrontamiento podría mitigar la violencia intrafamiliar y modificar su impacto en la salud mental.

Siendo así, es pertinente preguntarse, ¿Cuáles son las estrategias que utilizan las mujeres para afrontar la violencia ejercida por sus parejas? Akl Moanack (2016) toma la referida clasificación de Lazarus para su estudio de estrategias de afrontamiento en mujeres víctimas de violencia intrafamiliar, y las clasifica para su estudio en aquellas de tipo emocional y en aquellas focalizadas en el problema.

La autora encontró que las estrategias de afrontamiento más utilizadas son las de tipo emocional, ya que ante una situación de violencia, la víctima prefiere acudir a sus amigos y/o familia para recibir el apoyo moral que le permita reponerse del sufrimiento y poder continuar en convivencia con su agresor o, en un segundo momento, enfrentar activamente la problemática. 
Sin embargo el apoyo recibido de familiares y amigos generalmente no es suficiente motivación para llegar a utilizar estrategias externas, ya que el miedo ante la pérdida del apoyo económico, al cambio de la conformación familiar -especialmente cuando hay hijos-, y a la alteración del estatus social, tienen un efecto disuasorio que suele prevalecer.

Dada esta circunstancia, la autora concluye que las estrategias se convierten en elementos fundamentales para conseguir un cambio en las familias atravesadas por esta problemática.

\section{Estrategias de afrontamiento en Niños y adolescentes}

Transmisión de las estrategias de afrontamiento mediante la crianza

En general se ha señalado la existencia de una relación entre la crianza recibida por los niños y adolescentes y su utilización de estrategias de afrontamiento efectivas.

De alli la importancia de comprender los factores que contribuyen al desarrollo de diferentes modos de afrontamiento, especialmente para los niños en riesgo.

En su estudio, Smith (2019) efectivamente encuentra una relación causal entre la socialización parental y las estrategias y eficacia del afrontamiento de los niños.

Esta misma relación causal la encuentra Velez (2011) en un estudio, que referenciamos más adelante.

Así, para Smith, las prácticas parentales de socialización, tales como prestarle apoyo a los hijos o tener consistencia disciplinaria, se asociaron tanto con diferencias en el afrontamiento como en el ajuste de los niños. Agrega, además, que esta relación a veces es moderada por el consumo alcohólico de los padres. 


\section{Las estrategias más prevalentes en niños}

Ahora bien, ¿cuáles son las estrategias que los niños más utilizan?. E1 estudio de Rodríguez y Trianes (2016) permite conocer la prevalencia de estrategias de afrontamiento en tres problemas cotidianos infantiles familia, educación y salud- y correlacionándolas también con variables sociodemográficas, de contexto con evaluaciones de sus profesores. Para su estudio, refieren también a la clasificación de estrategias de Lazarus y llegan a la conclusión que en niños entre 8 y 13 años, -en general, sin diferenciar por ámbitos-, la estrategia más prevalente es la solución activa, seguida de la estrategia de Emoción. Estas estrategias, Solución activa y Emoción, forman parte de los dos principales estilos de afrontamiento propuestos en otros estudios.

Por otra parte, señalan que las estrategias de Evitación conductual y Pasividad aparecen como las menos prevalentes.

\section{Adolescentes, depresión y suicidio}

En Horwtiz (2011) podemos ver de qué manera las estrategias de afrontamiento usadas por los adolescentes pueden tener implicaciones para el desarrollo de depresión e ideación suicida. En su estudio, Horwtiz (2011) examinó las categorias de afrontamiento y los comportamientos de afrontamiento específicos usados por adolescentes; encontró que específicamente los comportamientos de desconexión conductual y auto culparse fueron predictivos de niveles más altos de depresión. A su vez la depresión y la recurrencia a estrategias de tipo emocional predijeron la ideación suicida.

Los resultados sugieren que los comportamientos específicos dentro de las amplias categorias de afrontamiento centradas en las emociones como por ejemplo Autoculparse y afrontamiento evitativo como 
Desconexión conductual, explican las asociaciones de estas categorias con la depresión y la ideación suicida.

Por otra parte las estrategias de afrontamiento centradas en el problema no predijeron de forma independiente niveles más bajos de depresión ni de ideación suicida.

\section{Estrategias de afrontamiento en el Divorcio}

Afrontamiento de los adultos frente al divorcio. El impacto de la dimensión espiritual

Amato (2000) sugiere que aquellos que se divorcian experimentan un incremento del estrés psicológico, tales como una mayor depresión y una reducción en su felicidad. Sin embargo, continúa, el impacto del divorcio varía de un individuo a otro y puede ser tanto positivo como negativo.

Partiendo de esa base, Krumrei (2011) muestra que el modelo de afrontamiento al estrés -en su dimensión espiritual- puede dar cuenta de algunas de estas diferencias y predecir el ajuste psicosocial de los adultos después del divorcio.

Los hallazgos específicos fueron que el tipo de afrontamiento religioso -"positivo": vgr. a través de la búsqueda espiritual o "negativo" vgr. valorar el divorcio como una profanación o un castigo divino-, significó la diferencia un año después entre un mayor crecimiento postraumático por un lado, o síntomas de depresión y persistencia de conflictos con el ex cónyuge por el otro. 
Efectos de los conflictos entre progenitores sobre los niños a largo plazo después del divorcio

Una vez producido el divorcio -que es un factor de riesgo para el desarrollo de psicopatologías que está bien documentado- surge la pregunta ¿Cómo operan las estrategias de afrontamiento de los niños cuando estos se ven expuestos al conflicto interparental post divorcio?

En su estudio para la Universidad de Cambridge, O’hara (2019), evalúa los potenciales efectos protectores del afrontamiento de los niños para mitigar ese riesgo a largo plazo.

El estudio encontró que los efectos del afrontamiento pueden predecir los resultados a largo plazo en relación a problemas mentales infantiles, uso de substancias y a conducta sexual arriesgada.

De esta manera el estudio apoya la noción de que mejorar la capacidad general de la juventud para afrontar adaptativamente es un factor protector potencialmente modificable para todos los niños que enfrentan el divorcio parental.

\section{Proteger a los niños de los efectos del divorcio}

Efectos de la paternidad en los procesos de afrontamiento de los niños

Habiendo determinado la relación entre afrontamiento y psicopatología de los niños frente al divorcio, ¿Cómo puede protegerse a los niños de los efectos del divorcio? O en términos del estudio de O`hara, ¿Cómo mejorar esa capacidad para afrontar adaptativamente?

Una manera posible es trabajo psicoeducativo con los padres, puesto que, como dijimos, para Vélez (2011) existe una fuerte relación causal entre la crianza y los procesos de afrontamiento de los niños. 
En el estudio referenciado, Vélez examina si los cambios inducidos por las intervenciones sobre la calidad de la relación madre-hijo condujeron a cambios en los procesos de afrontamiento de los niños.

Los análisis revelaron que efectivamente fue así, las mejoras inducidas en la relación madre-hijo condujeron a aumentos en la eficacia de afrontamiento a corto plazo, a los 6 meses, y a aumentos en la eficacia de afrontamiento y afrontamiento activo en el largo plazo, hasta 6 años.

Este hallazgo permite inferencias causales más fuertes sobre las relaciones entre la crianza y los procesos de afrontamiento de los niños, identificando la calidad de la relación madre-hijo como un importante predictor -modificable- de los procesos de afrontamiento de los niños.

El contacto con los abuelos como factor de protección

Siguiendo con los niños que enfrentan el divorcio de sus padres, otro vector importante a considerar en las intervenciones es fortalecer el contacto con los abuelos. En apoyo de esta idea, Sorek (2020), estudio la calidad de vida de hijos de padres divorciados y relacionó el factor de riesgo "conflicto parental" con factores de resiliencia sociales tales como apoyo social general percibido, cercanía a los abuelos y discurso abierto con ellos sobre el divorcio.

Estudiando esa relación, Sorek llego a la conclusión de que el conflicto de los padres estaba asociado negativamente a la calidad de vida de los niños, y en general el apoyo y la relación cercana con los abuelos se asociaron positivamente a la misma. 


\section{Conclusiones}

Los acontecimientos estresantes alteran el equilibrio dinámico familiar, de ahí la importancia de que la familia posea buenas estrategias de afrontamiento, pues la evidencia empírica reunida en el artículo señala en esa dirección: las estrategias de afrontamiento median entre los estresores y la salud de la familia como grupo y de sus integrantes individualmente.

La Comprensión de los vínculos entre factores que son potencialmente modificables y los procesos de afrontamiento resulta clave para el diseño de intervenciones para poblaciones en riesgo que están expuestos a niveles elevados de factores estresantes, como las parejas en conflicto o atravesando un proceso de divorcio, y sus hijos, sean niños o adolescentes.

Por otro lado, es importante que los profesionales de la salud conozcan los estresores específicos así como las estrategias de afrontamiento familiar positivas, para que, mediante la promoción de la salud, puedan prevenirse los problemas derivados de un inadecuado afrontamiento familiar. 


\section{Referencias}

Akl Moanack, P. M., Pilar Jiménez, E., \& Lucía Aponte, F. (2016). Estrategias de afrontamiento en mujeres víctimas de violencia intrafamiliar. Cultura, educación y sociedad $7(2), 105-121$.

Amato, P. R. (2000). The consequences of divorce for adults and children. Journal of Marriage \& the Family, 62, 1269-1287.doi:10.1111/j.1741-3737.2000.01269.x

Compas, B. E., Connor-Smith, J. K., Saltzman, H., Thomsen, A. H., \& Wadsworth, M. E. (2001). Coping with stress during childhood and adolescence: Problems progress and potential in theory and research. Psychological Bulletin, 127, 87-127

Goleman, D. (1995). La inteligencia emocional. Buenos Aires. Ed. Javier Vergara Editor

Horwitz, A. G., Hill, R. M., \& King, C. A. (2011). Specific coping behaviors in relation to adolescent depression and suicidal ideation. Journal of adolescence, 34(5), 1077-1085. Doi: https://doi.org/10.1016/j.adolescence.2010.10.004

Krumrei, E. J., Mahoney, A., \& Pargament, K. I. (2011). Spiritual stress and coping model of divorce: A longitudinal study. Journal of family psychology, 25(6), 973. https://doi.org/10.1037/a0025879

Lazarus, R. S. (1966). Psychological stress and the coping process. New York, NY: McGrawHill.

Lazarus, R. S., \& Folkman, R. S. (1984). Stress appraisal and coping. New York, NY: Springer Publishing Company.

Martínez-Montilla, J. M., Amador-Marín, B., \& Guerra-Martín, M. D. (2017). Estrategias de afrontamiento familiar y repercusiones en la salud familiar: Una revisión de la literatura. Enfermeria Global, 16(47), 576-604. http://dx.doi.org/10.6018/eglobal.16.3.255721

Mengo C., Small E., \& Black B. (2017). Intimate Partner Violence and Women's Mental Health: The Mediating Role of Coping Strategies Among Women Seeking Help From the Police. Journal of Interpersonal Violence. doi:10.1177/0886260517729402

Morales Rodriguez F.M., Trianes Torres M.V., \& Miranda Páez J. (2016). Prevalence of strategies for coping with daily stress in children. Psicothema. 2016 28(4),370-376. doi: $10.7334 /$ psicothema2015.10

O'Hara, K. L., Sandler, I. N., Wolchik, S. A., \& Tein, J. Y. (2019). Coping in context: The effects of long-term relations between interparental conflict and coping on the development of child psychopathology following parental divorce. Development and 
psychopathology, 31(5), 1695-1713. DOI:

https://doi.org/10.1017/S0954579419000981

Papp, L. M., \& Witt, N. L. (2010). Romantic partners' individual coping strategies and dyadic coping: Implications for relationship functioning. Journal of Family Psychology, 24(5), 551-559. https://doi.org/10.1037/a0020836

Smith, C. L., Eisenberg, N., Spinrad, T. L., Chassin, L., Morris, A. S., Kupfer, A. \& Kwok, O. M. (2006). Children's coping strategies and coping efficacy: Relations to parent socialization, child adjustment, and familial alcoholism. Development and Psychopathology, 18(2), 445-469. DOI: https://doi.org/10.1017/S095457940606024X

Sorek, Y. (2020). Grandparental and overall social support as resilience factors in coping with parental conflict among children of divorce. Children and Youth Services Review, 118,105443. https://doi.org/10.1016/j.childyouth.2020.105443

Vélez, C. E., Wolchik, S. A., Tein, J. Y., \& Sandler, I. (2011). Protecting children from the consequences of divorce: A longitudinal study of the effects of parenting on children's coping processes. Child development, 82(1), 244-257. https://doi.org/10.1111/j.14678624.2010.01553. 